\title{
Block-Matching Distortion Correction of Echo-Planar Images with Opposite Phase Encoding Directions
}

\author{
Renaud Hédouin*, Olivier Commowick*, Elise Bannier* ${ }^{\ddagger}$, Benoit Scherrer ${ }^{\dagger}$, Maxime Taquet ${ }^{\dagger}$, \\ Simon K. Warfield ${ }^{\dagger}$, Senior Member IEEE Christian Barillot*, Senior Member IEEE \\ * INRIA Rennes - Bretagne Atlantique, VISAGES Research Team \\ Campus de Beaulieu, 35000 Rennes, FRANCE \\ $\dagger$ Computational Radiology Laboratory, Department of Radiology, \\ Children's Hospital, 300 Longwood Avenue, Boston, MA, 02115, USA \\ $\ddagger$ Department of Radiology, University Hospital of Rennes, Rennes, France
}

\begin{abstract}
By shortening the acquisition time of MRI, Echo Planar Imaging (EPI) enables the acquisition of a large number of images in a short time, compatible with clinical constraints as required for diffusion or functional MRI. However such images are subject to large, local distortions disrupting their correspondence with the underlying anatomy. The correction of those distortions is an open problem, especially in regions where large deformations occur.

We propose a new block-matching registration method to perform EPI distortion correction based on the acquisition of two EPI with opposite phase encoding directions (PED). It relies on new transformations between blocks adapted to the EPI distortion model, and on an adapted optimization scheme to ensure an opposite symmetric transformation. We present qualitative and quantitative results of the block-matching correction using different metrics on a phantom dataset and on in-vivo data. We show the ability of the blockmatching to robustly correct EPI distortion even in strongly affected areas.
\end{abstract}

Index Terms - Block-Matching, Echo Planar Imaging, Registration, Diffusion MRI

\section{INTRODUCTION}

In recent years, single-shot Echo Planar Imaging (EPI) has been increasingly used as it is substantially faster than most other acquisition sequences. The high speed of this acquisition comes from the fact that the image is acquired within a single-shot instead of multiple shots (single or multiple echoes) in other classical sequences (Gradient Echo, Spin Echo...). In regard of the required relaxation time between each shot, the single-shot method saves a considerable acquisition time. By shortening the acquisition time of every single time frame, EPI enables the acquisition of a larger number of images than other methods while respecting the same clinical constraint. This is particularly useful for diffusion-weighted imaging (DWI) wherein the acquisition of several scalar images is required to represent the underlying microstructure of the brain (white matter mainly) [1], [2], [3], [4]. For this reason, EPI is the most common sequence used for DWI [5]. For similar speed reasons, EPI is also used for functional imaging [6], which requires the fast acquisition of many brain images while a task is executed by the subject.

The high velocity of EPI acquisitions comes at the cost of a high sensitivity to magnetic field inhomogeneities. Affected areas, often located at the tissue interfaces with different magnetic susceptibilities such as bone or air, are either contracted or dilated along the phase encoding direction (PED) [7] (moreover, measured tissue intensities in these regions change due to the local transformation). Therefore the brain anatomy in EPI does not match with structural images that are much less sensitive to distortions. Such a correspondence is however critical as a joint analysis is often performed for these modalities: 1for diffusion imaging, structural images are used to define regions of interest for fiber tracking or to extract lesions that are to be linked to brain microstructure properties ; 2- for functional MRI, activations are computed on low resolution EPI to speed up acquisition time and need to be aligned with a high resolution $\mathrm{T} 1 \mathrm{w}$ image at least for interpretation and visualization of activated regions in the brain. In both cases, it is therefore necessary to perform EPI distortion correction as non linear anatomy mismatch between the modalities will lead to biased results. Computing such a distortion correction is still an open problem, especially in regions where large deformations occur.

As the distortion in EPI acquisitions comes from the $B_{0}$ field inhomogeneities, the first technique for distortion correction relies on the acquisition of a $B_{0}$ field map [7], [8]. This map is in turn used to infer the local contractions and dilations, and correct EPI intensities. This field map however needs to be smoothed to avoid noise corruption and may therefore be unable to provide sufficient correction in severely distorted areas [9], [10].

Other techniques have considered new sequences using point spread functions to obtain acquisitions with no distortion. This category includes works by Robson et al. [11], Chung et al. [12] and Zaitsev et al. [13]. Unfortunately such sequences are not currently available on all scanners.

A third class of methods considers the acquisition of two EPI sequences with opposite phase encoding directions one anterior-posterior and one posterior-anterior for exam- 
ple - to correct for distortion. This class of techniques, initially proposed by Chang and Fitzpatrick [14] and Bowtell et al. [15], relies on the computation of a distortion field from the two images to correct the EPI. Several methods use this technique: Andersson et al. [16] used a pair of reversed EPI in conjunction with a discrete model of image formation for spin-echo EPI. An implementation called TOPUP is available in the FSL package ${ }^{1}$. Voss et al. [17] introduced an algorithm to estimate, from the two images, the correction displacement field based on cumulative intensity distributions along each line in the PED. This simple method strongly reduces the distortion, however it is sensitive to noise and the computed transformation needs to be smoothed, leading to a trade-off between regularity and precision. Other methods in this category include Morgan et al. approach [18], using continuously alternating phase encoding, Weiskopf et al. method [19] using a modified multi-echo EPI acquisition with reversed phases, or Holland et al. algorithm [9] which performs an intensity-based registration (each line being considered independently). As for Voss et al. algorithm, the obtained displacement field is sensitive to noise, especially when large displacements are present. More recently a new method has been proposed to combine EPI with opposite PED with Point Spread Functions (PSF) [20] however costing additional acquisition time. Finally a registrationbased method has been proposed by Irfanoglu et al. [21] requiring a non distorted image such as a $\mathrm{T} 2$ image (in addition to the reversed PED image) which is used as the central point where the two images with reversed PEDs are transformed. It minimizes a cost function to compute a transformation which has no a priori restriction with respect to EPI image formation. The transformation is instead projected after each step of the minimization to follow a distortion model (with distortions appearing uniquely along the PED).

This last category of techniques has the advantage of requiring only a short additional acquisition time to correct for distortion: if we assume no patient movement occurred during the acquisition and that the magnetic field inhomogeneity stays constant during the acquisition [22], only one supplemental EPI image with reversed PED is necessary to correct the entire EPI series (e.g. fMRI or DWI acquisition). We therefore present a new algorithm for distortion correction falling in the same category. Block-matching based registration has been successfully proposed for registration in medical imaging both for rigid [23] and non-linear registration [24]. As a registration framework, block-matching has the advantage of being very generic and easily adaptable to different transformation priors, both to match blocks in the floating image [25] and for the global transformation (linear or non-linear). Moreover, this framework is also robust to outliers in the local matches. We therefore propose in this work a new approach towards distortion correction of EPI based on block-matching. It is designed to register two images ac-

\footnotetext{
${ }^{1}$ http://fsl.fmrib.ox.ac.uk/fsl/fslwiki
}

quired with opposite $\mathrm{PED}$ without requiring an additional structural image. To do so, we introduce a new symmetric block-matching registration algorithm, optimizing local affine transformations constrained a priori in the PED to match the expected distortions in EPI. In addition, the transformation is computed as opposite symmetric to match the distortion model in EPI [7]. The implementation of our algorithm is available in our open source medical image processing toolbox Anima ${ }^{2}$.

We evaluate our new algorithm qualitatively and quantitatively on two datasets in Section IV. First, we present results on EPI acquisitions of a phantom, where the geometry of the image is known. We also perform evaluation on in-vivo diffusion-weighted EPI of five subjects for which images with four different PED (anterior-posterior (AP), posterior-anterior (PA), left-right (LR), right-left $(\mathrm{RL})$ ) were acquired. We present our results in contrast to two state-of-the-art methods using the same inputs: TOPUP from Andersson et al. [16] and Voss et al. method [17]. Results demonstrate the ability of our algorithm to compute a robust EPI distortion correction.

\section{METHODS}

\section{A. Distortion Model}

We assume that two images have been acquired: $I_{F}$ is the EPI forward image acquired with a classical PED (AP for example), and $I_{B}$ is the EPI backward image acquired with a reversed PED (PA in this case). The goal of EPI distortion correction is to estimate a distortion transformation field from these two images. Then, from this field, it is possible to recover a corrected image $C$ from these two images, but also an entire serie of EPI acquired with AP or PA PED. Jezzard et al. [7] have demonstrated that deformations due to $B_{0}$ field inhomogeneities appear mainly along the PED and are negligible in other directions. More precisely, we follow the distortion model as expressed previously in [17], [18] which assumes that $I_{F}$ and $I_{B}$ are generated from the theoretical corrected image $C$ using a displacement field parallel to the PED:

$$
\begin{cases}C(x)= & J_{T_{+}}(x) I_{F}\left(T_{+}(x)\right) \\ C(x)= & J_{T_{-}}(x) I_{B}\left(T_{-}(x)\right)\end{cases}
$$

where $T_{+}(x)=x+U(x)$ and $T_{-}(x)=x-U(x) . J_{T_{+}}$ and $J_{T_{-}}$denote the Jacobian determinants of the local deformations which account for intensity changes in the distorted areas. It will lead to an increased intensity in the contracted areas and a decreased intensity in the dilated areas. $U$ corresponds to the distortion displacement field which is parallel to the PED, e.g. if the PED is along the y-axis then $U(x)=\left[\begin{array}{lll}0 & U_{y}(x) & 0\end{array}\right]^{T}$. It is assumed in this model that $T_{+}$and $T_{-}$are opposite symmetric, i.e. that they share the same $U$ up to a minus sign along the PED.

\section{B. Block-Matching for Distortion Correction}

Different approaches may be considered to match the two images. In the distortion model, the corrected image

\footnotetext{
${ }^{2}$ https://github.com/Inria-Visages/Anima-Public
} 
$C$ is generally unknown. It could be replaced (as suggested in [21]) by a non distorted similar acquisition (such as a T2 weighted acquisition). However, this is not always available in clinical acquisitions. We therefore consider the case where $C$ can be at best estimated and choose a registration approach that does not rely on it. A registration method has been introduced by Avants et al. [26] allowing to estimate the corrected image $C$ without having it directly appear in the algorithm. The idea, instead of looking for the transformation $T$ between two images, is to seek the half-transformation $T^{1 / 2}$ so that the two images registered from $I_{F}$ and $I_{B}$ match as much as possible:

$$
I_{F} \circ T^{1 / 2} \approx I_{B} \circ T^{-1 / 2} \approx C
$$

We adapt this approach to a block-matching algorithm [23], [24] by constraining the transformation to be aligned with the PED as assumed in the distortion model. The block-matching algorithm enables a simple and effective incorporation of this constraint on the deformation field. First we present the global scheme of the block-matching algorithm, then we detail each part separately. We consider an initial transformation $U_{0}$ which can be null or coming from another coarse correction algorithm. We use a classic multi-resolution pyramidal scheme [27] to process images from coarse to fine resolution. At each level of the pyramid, from the transformation at the previous pyramid level, we proceed as described in Algorithm 1 and illustrated as a diagram in Fig. 1.

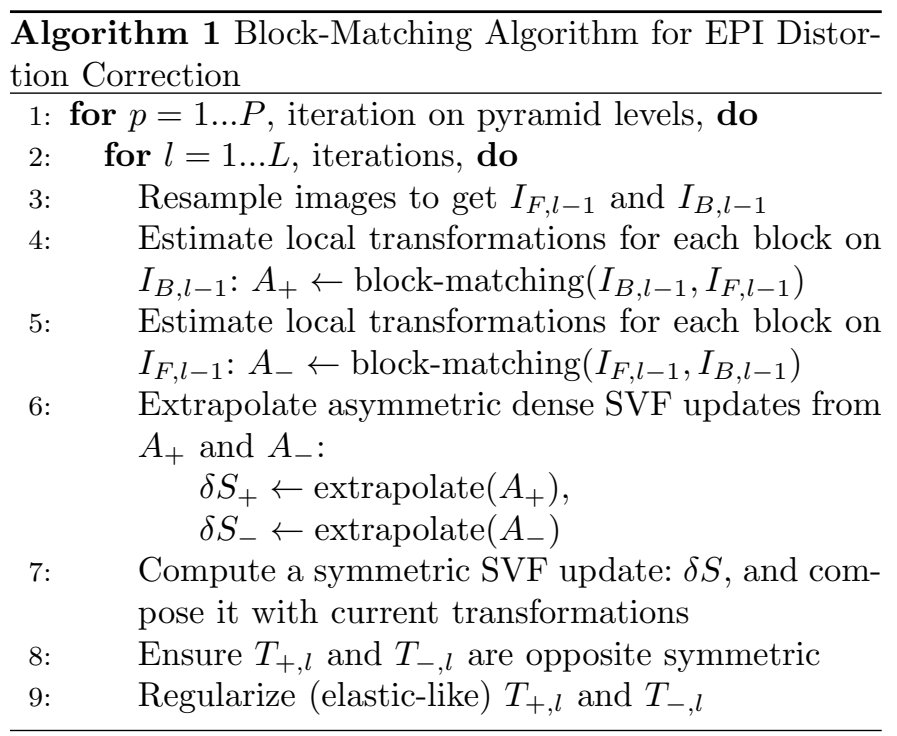

At each step, we first resample the original images with the current transformation. Then we estimate pairings between the images in the forward and backward directions $\left(A_{+}=\left\{\hat{A}_{+, 1}, . ., \hat{A}_{+, N}\right\}\right.$ and $\left.A_{-}=\left\{\hat{A}_{-, 1}, . ., \hat{A}_{-, N}\right\}\right)$ using a block-matching algorithm. We utilize $A_{+}$and $A_{-}$to extrapolate two asymmetric stationary velocity fields (SVF) $\delta S_{+}$and $\delta S_{-}$which are combined into a symmetric SVF update $\delta S$ (Section II-C). We then compose this update with $T_{+, l-1}$ and $T_{-, l-1}$, and ensure that $T_{+}$and $T_{-}$still share the same displacement field $U$. Finally, the current displacement field is regularized using a convolution with a Gaussian kernel (standard deviation $\sigma_{E}$ ). The following sections detail the block-matching, extrapolation and composition steps of this algorithm and their specificities for distortion correction of EPI.

1) Block-Matching of Distorted EPI: At each iteration, we define blocks $B_{+, i}$, which are patches centered at $x_{i}$ with size $(2 N+1)^{3}$, regularly placed on image $I_{B, l-1}$ (every $V$ voxels in each direction). We also define blocks $B_{-, i}$ with the same characteristics on $I_{F, l-1}$. For each of those blocks, we look for an adapted transformation best matching them respectively to $I_{F, l-1}$ and $I_{B, l-1}$. Let $\mathcal{L}$ be the set of allowed transformations for matching blocks. Frequently, in other applications, the transformation sought between blocks is a simple 3-dimensional translation. In the case of EPI distortion, the set $\mathcal{L}$ can be further adapted to match a priori the expected features of the distortion at the block level and thus obtain a more robust transformation estimation. First the model assumes that distortions appear uniquely along the PED: a one-dimensional translation along the PED (modeled by one parameter $t_{., i}$ ) is therefore sufficient. At the scale of the block, a single translation is however not enough to account for local contractions and dilations due to the distortion at different points of the block. We account for this by adding three parameters to the transformation. The first one accounts for the change of scale due to the global contraction or dilation inside the block $\left(s_{., i}\right)$. This scale parameter solves the problem of global scaling inside the block, however different lines along the PED will have different distortions generating skewness at the block level. To consider this, we define two skew components $\left(k_{., i}\right.$ and $\left.m_{., i}\right)$ for the two directions complementary to the PED. Assuming the PED is the yaxis, $A_{., i}$ can be expressed as a $4 \times 4$ matrix:

$$
A_{., i}=\left(\begin{array}{cccc}
1 & 0 & 0 & 0 \\
k_{., i} & s_{., i} & m_{., i} & t_{., i} \\
0 & 0 & 1 & 0 \\
0 & 0 & 0 & 1
\end{array}\right)
$$

Note that having the PED on an other axis will result in the line of parameters being displaced on the first or third line of the matrix. A few interesting properties are associated to this transformation. The Jacobian determinant of the transformation is simply computed as the scaling parameter $s_{., i}$, and this parameter is therefore utilized directly for modeling the intensity changes due to distortion at the block level. In addition, this transformation has an analytic form for its matrix logarithm, and may therefore be directly estimated in the log-Euclidean space to speed up the global SVF extrapolation process (see Section II-C). The block-matching step then amounts to estimate the four log-parameters of each block transformation in $\mathcal{L}$ to compute the set of optimal transformations $\hat{A}_{+, i}$ and $\hat{A}_{-, i}$ (respectively for blocks $B_{+, i}$ and $B_{-, i}$ ) optimizing a similarity measure $S$ between $I_{F, l-1}$ and $I_{B, l-1}$ : 
Symmetrize the transform Go back to the regular space

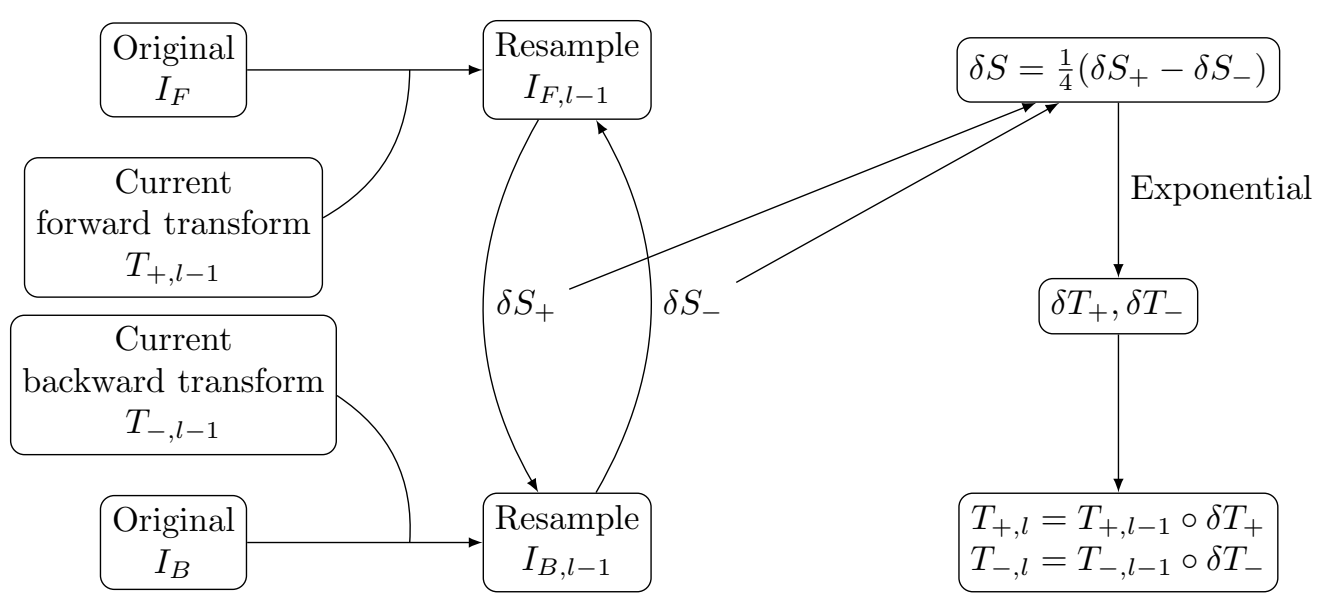

Fig. 1. Graphical illustration of one iteration of the block-matching algorithm.

$$
\begin{aligned}
& \hat{A}_{+, i}=\underset{A_{+, i}}{\arg \max } S\left(J_{A_{+, i}} I_{F, l-1} \circ A_{+, i}, I_{B, l-1}\right) \\
& \hat{A}_{-, i}=\underset{A_{-, i}}{\arg \max } S\left(I_{F, l-1}, J_{A_{-, i}} I_{B, l-1} \circ A_{-, i}\right)
\end{aligned}
$$

This optimization is performed using the BOBYQA algorithm for gradient free optimization with parameters within predefined bounds [28]. It proceeds by successively computing quadratic approximations to the cost function to find its local maximum.

2) Confidence weights: We have computed for each block $B_{., i}$ the local transformation $\hat{A}_{., i}$ that optimizes the similarity measure $S$. We then assign to this transformation a weight $w_{., i}$ to estimate the confidence in the block match. To do so, we use a combination of two different terms. The first one is the similarity itself $\hat{S}_{., i}$ assuming it belongs to the range $[0,1], 1$ being the best result (if not, a function of $\hat{S}_{., i}$ can be used). The second one, $w_{d}\left(B_{., i}\right)$ gives an index of the local structure of the reference block along the PED. If the block structure is parallel to the PED, all tested transformations $A_{., i}$ for that block may get the same similarity score, thereby introducing uncertainty in the matches. Actually, the algorithm will always find a solution due to small variations of intensities or computing precision. However the quality of such solutions will be random. We therefore want to avoid as much as possible such random solutions which can propagate important errors. Only considering the optimal similarity is therefore not enough and we introduce the index $w_{d}$ to give a low weight to these uncertain blocks and their corresponding local transformations. $w_{d}$ is defined as a function of the structure tensor inside the reference block $B_{., i}$ :

$$
w_{d}\left(B_{., i}\right)=c_{l}\left(D_{B_{., i}}\right)<\hat{v}_{D_{B_{., i}}}, g>
$$

- $D_{B_{., i}}$ is the average structure tensor of block $B_{., i}$
- $c_{l}\left(D_{B_{., i}}\right)$ is a linear coefficient which quantifies the anisotropy of the tensor [29]

$$
\begin{aligned}
- & c_{l}\left(D_{B_{., i}}\right)=\frac{\lambda_{1}-\lambda_{2}}{\lambda_{1}} \text {, with } \lambda_{1} \geqslant \lambda_{2} \geqslant \lambda_{3} \text { the } \\
& \text { eigenvalues of } D_{B(., i)}^{n_{(1, i)}}
\end{aligned}
$$

- $c_{l}\left(D_{B_{., i}}\right)$ is close to 0 if $D_{B_{., i}}$ has a planar or circular structure and close to 1 if it has a very anisotropic one

- $\hat{v}_{D_{B}, i}$ is the principal eigenvector of $D_{B_{., i}}$

- $g$ is the unit vector along the PED

$w_{d}$ will be 0 if the structure tensor is perpendicular to the PED (image structure parallel to $g$ ) or planar/spherical $\left(c_{l}\right.$ $=0$ ) giving a structure based confidence to the matches. Finally, the weight for the match of block $B_{., i}$ is defined as the geometric mean of the similarity index and the structural index:

$$
w_{., i}=\sqrt{w_{d}\left(B_{., i}\right) \hat{S}_{., i}}
$$

\section{Transformation Extrapolation and Composition}

From the block-matching algorithm, two sets of block pairings have been constituted: one for $I_{F}, \hat{A}_{+}=$ $\left(\hat{A}_{+, 1}, \ldots, \hat{A}_{+, m}\right)$, and one for $I_{B}, \hat{A}_{-}=\left(\hat{A}_{-, 1}, \ldots, \hat{A}_{-, n}\right)$. Each pairing is defined by the center of its corresponding block $B_{., i}$, a transformation $\hat{A}_{., i}$, and a weight $w_{., i}$. We then extrapolate two update SVFs from the sparse weighted transformation logarithms: $\delta S_{+}=$ extrapolate $\left(\hat{A}_{+}\right)$and $\delta S_{-}=\operatorname{extrapolate}\left(\hat{A}_{-}\right)$. This extrapolation aims at computing a dense field of transformation logarithms $\hat{R}_{., i}(i=1, \ldots, M$ representing each voxel) from the sparse $\hat{A}_{., j}$. This is performed utilizing an Msmoothing algorithm in the log-Euclidean space on affine transformations [30] as proposed in [24]: 


$$
\begin{aligned}
& \left(\hat{R}_{., 1}, \ldots, \hat{R}_{., M}\right)=\underset{R_{., 1}, \ldots, R_{., M}}{\arg \min } \\
& \sum_{i=1}^{M} \sum_{j \in \mathcal{N}_{i}} w_{B,, j} \rho\left(\left\|R_{., i}-\log \hat{A}_{\cdot, j}\right\|^{2}\right) d\left(\left|x_{i}-x_{j}\right|^{2}\right)
\end{aligned}
$$

where log denotes the matrix logarithm, $x_{j}$ is the spatial position of pairing, $\mathcal{N}_{i}$ is the neighbourhood of $x_{i}, \rho$ is a robust error norm to account for outliers in the set of sparse transformations $\hat{A}$. (here the Welsh function), $d$ is a function of the Euclidean distance - here $d\left(b^{2}\right)=$ $\exp \left(-b^{2} / 2 \theta^{2}\right)$ - giving more importance to spatially close reference pairings. This cost function is optimized through an iterative scheme, more detailed in [24]. The obtained transformation logarithms $\hat{R}_{., i}$ are then applied to their respective positions $x_{i}$ to compute the SVFs $\delta S_{+}$and $\delta S_{-}$: $\delta S .\left(x_{i}\right)=\hat{R}_{., i} x_{i}$. Extrapolating update SVFs using this Msmoothing algorithm, we incorporate an outlier rejection framework, mainly coming from the $\rho$ function in Eq. (8), that removes from the resulting SVF erroneous block transformation logarithms due for example to artifacts or other effects in $I_{F, l-1}$ and $I_{B, l-1}$. In addition, the extrapolated fields are SVFs and therefore encode diffeomorphic and invertible transformations.

A symmetric $\delta S$ is then computed, following Vercauteren et al. approach [31], as a quarter of the subtraction of asymmetric incremental updates $\delta S_{+}$and $\delta S_{-}$:

$$
\delta S=\frac{1}{4}\left(\delta S_{+}-\delta S_{-}\right)
$$

While the half difference of the two asymmetric incremental updates is sufficient to compute a symmetrized field, $\delta S$ is computed as a quarter of the difference as we are seeking the transformation bringing the two input images towards an unknown middle image $C$. As such only the half symmetric SVF is needed.

The final step of each iteration then amounts to composing the updates with the current transformations and ensure that the resulting $T_{+, l}$ and $T_{-, l}$ transformations still share the same displacement field $U$ at the $l$-th iteration $U_{l}$. To do so, $\delta T_{+}$and $\delta T_{-}$are first obtained by exponentiating $\delta S: \delta T_{+}=\exp (\delta S)$ and $\delta T_{-}=\exp (-\delta S)$ [30], and composed with the current transformations: $T_{+, l}=T_{+, l-1} \circ \delta T_{+}$and $T_{-, l}=T_{-, l-1} \circ \delta T_{-}$. As the composition and inverse operations do not ensure the opposite symmetry condition, we finally compute the shared displacement field $U_{l}$ as $U_{l}(x)=\frac{1}{2}\left(T_{+, l}(x)-T_{-, l}(x)\right)$ such that $T_{+, l}(x)=x+U_{l}(x)$ and $T_{-, l}(x)=x-U_{l}(x)$.

\section{Experimental Design}

\section{A. Image acquisitions}

Imaging was performed on a Siemens Verio 3T scanner. The approach was evaluated on in vitro and in vivo data:

- In vitro: A grid phantom with known geometry developed by the UNIRS group from the CEA Neurospin for the CATI Consortium for image acquisition and processing $^{3}$ was imaged : $b_{0}$ images (AP, PA, LR and $\mathrm{RL}$ ) were acquired with a 12 channels coil, a $128 \times 128 \times 60$ matrix size and a $2 \times 2 \times 2 \mathrm{~mm}^{3}$ voxel size.

- In vivo: 5 healthy volunteers were imaged after approval from the local institutional review board. For each volunteer, two pairs of $b_{0}$ EPI images with opposite PED (AP/PA and LR/RL), $128 \times 128 \times 60$ matrix size and $2 \times 2 \times 2 \mathrm{~mm}^{3}$ voxel size were acquired with a 32 channels head coil. The EPI images were acquired using the parallel imaging method GRAPPA with an acceleration factor of $2(T E=82 \mathrm{~ms}$ and an echo space $0.69 \mathrm{~ms}$ ). Regular clinical DWI were also acquired (30 gradient directions, $b=1000$ s. $\mathrm{mm}^{-2}$ ) with identical geometry and AP PED.

\section{B. Experimental methods}

1) Evaluation strategy: In order to estimate the quality of the distortion correction we follow the process illustrated in Fig. 2. We first estimate a corrected image $C_{A P / P A}$ from one pair of reversed $b_{0} \mathrm{EPI}(\mathrm{AP} / \mathrm{PA})$. Then, independently, we estimate a corrected image $C_{L R / R L}$ from the other pair of reversed $b_{0} \mathrm{EPI}(\mathrm{LR} / \mathrm{RL})$. Each pair of reversed EPI has a PED following the same orientation but with an opposite direction. However the two pairs have a PED following a different orientation. This means that distortions will affect different areas of the brain in a different way depending on the orientation of the PED. We thus consider the quality of the match between $C_{A P / P A}$ and $C_{L R / R L}$ as an index to estimate the general quality of the distortion correction (see Fig. 2). If the two pairs of images are perfectly corrected, they will match after correction. In the following, we compare three different distortion correction methods with different metrics.

2) Method 1: Voss: The first evaluated method was proposed by Voss et al [17]. Their approach amounts, for each line in the PED, for both images, to do:

- Compute normalized cumulated intensities

$$
N_{i}(y)=\frac{1}{\alpha_{i}} \int_{0}^{y} L_{i}(x) \mathrm{d} x \quad \text { for } i=1,2
$$

- $L_{1}$ and $L_{2}$ are line intensities of images $I_{F}$ and $I_{B}$, $\alpha_{1}$ and $\alpha_{2}$ are normalization constants:

$$
\alpha_{i}=\int_{0}^{\infty} L_{i}(x) \mathrm{d} x \quad \text { for } i=1,2
$$

- For a large number $n$ of values $x_{n}$ between 0 and 1 , find by cubic interpolation [32] $y_{1, n}$ and $y_{2, n}$ such that $N_{1}\left(y_{1, n}\right)=N_{2}\left(y_{2, n}\right)=x_{n}$

- At each position $y_{n}=\left(y_{1, n}+y_{2, n}\right) / 2$, the transformation map is computed as

$$
U\left(y_{n}\right)=y_{1, n}-y_{n}=y_{n}-y_{2, n}
$$

This algorithm has the advantage of being very fast and simple. However, it is highly sensitive to noise and lines registrations are purely independent which may lead to unrealistic transitions between consecutive lines. Therefore,

\footnotetext{
${ }^{3}$ http: //cati-neuroimaging.com
} 
Correction from AP-PA images $\quad$ Correction from LR-RL images
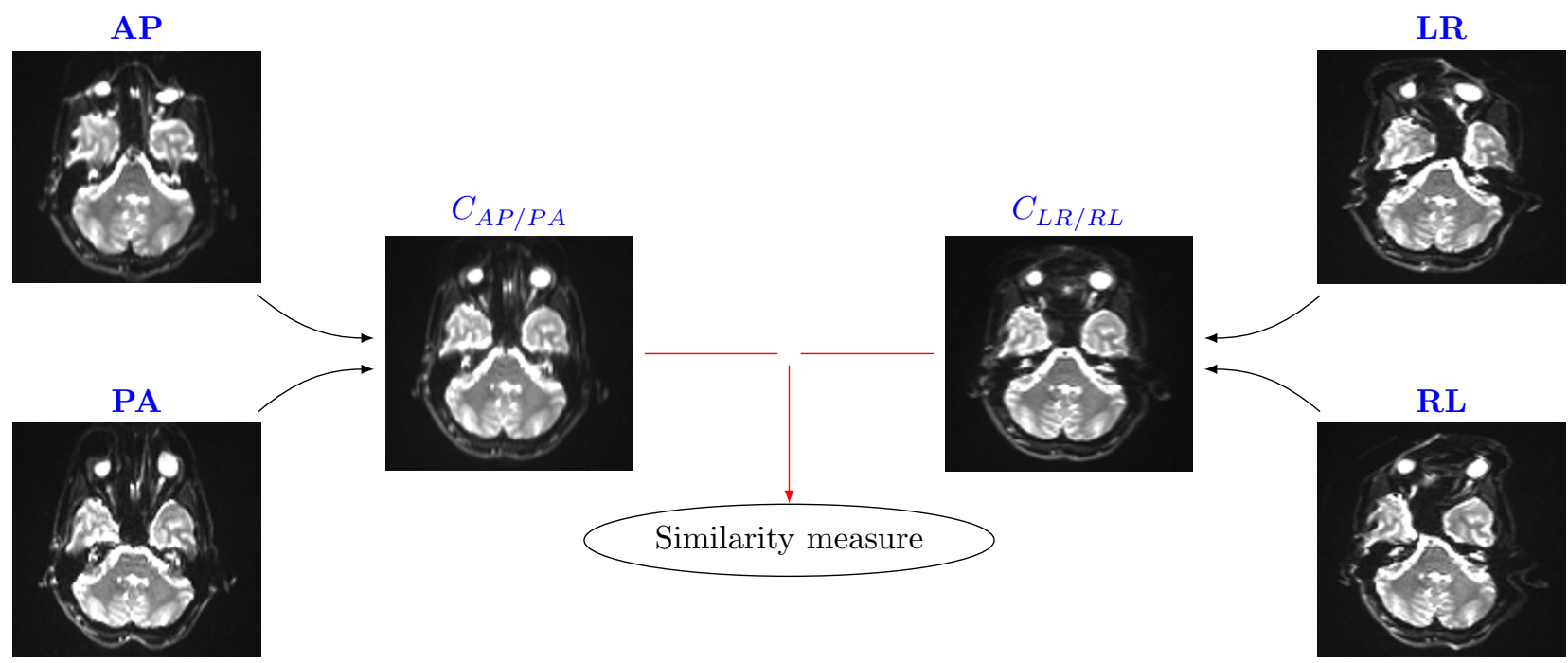

Fig. 2. Illustration of the evaluation process for two pairs of EPI images with different reversed PEDs. On the left side, the AP (Top) and the PA (Bottom) images corrected by BM (Middle). On the right side, the LR (Top) and the RL (Bottom) images corrected by BM (Middle). A similarity measure between the 2 corrected images is performed to estimate the quality of the corrections.

a 3D Gaussian smoothing with a standard deviation $\sigma$ is performed on the obtained transformation $T$, which leads to a trade-off between transformation precision (small Gaussian $\sigma$ ) and transformation regularity (high Gaussian $\sigma)$. In our experimentations, we have set $\sigma$ to one pixel.

3) Method 2: TOPUP: In addition to Voss et al. method, we also evaluated the distortion correction obtained from the TOPUP algorithm, available within the FSL package ${ }^{4}$. This correction method is based on the work from Andersson et al. [16]. It uses a model of EPI image formation and, together with the two images with opposite PEDs, reconstructs a deformation field to obtain a corrected EPI. More details are provided in Andersson et al. publication or on the FSL documentation page. We utilized the default parameters of this method for all of our experiments.

4) Method 3: Proposed block-matching technique: Distortion correction involves finding very large and local displacements between the images, displacements that may be extremely difficult to recover for registration approaches. We tackle this problem using a coarse-to-fine approach to recover EPI distortion and using Voss et al. method with a large $\sigma$ smoothing value as an initial transformation, which is then further improved with our block-matching strategy.

Aside from transformation initialization, the blockmatching implementation has different parameters, that are set as follows. First, we use three levels on the multiresolution pyramid and 10 iterations at each level. The size of each block is $3 \times 3 \times 3$ (i.e. $N=1$ ). These blocks are placed regularly every two voxels in each direction $(V=2)$. The initial search radius for BOBYQA is set to 2 voxels, the initial skew radius is set to 0.1 , and the

${ }^{4}$ http://fsl.fmrib.ox.ac.uk/fsl/fslwiki/TOPUP initial scale radius to 0.1 . We use a squared correlation coefficient as the similarity measure between blocks. At the end of each iteration, the elastic regularization is made with a $\sigma_{E}$ value of 2 voxels. These parameters were set to the same values for each distortion correction experiment.

\section{Evaluation Metrics}

1) Phantom Evaluation: point-based metric: Contrary to brain images, the phantom acquisition has a known grid structure on which landmarks are easily identifiable. For each uncorrected image (AP, PA, LR and RL), 20 landmarks were carefully and manually selected at voxels representing crossing points. To evaluate quantitatively distortion correction, we have then applied the following steps for each evaluated method. First, images AP and PA, respectively LR and RL, were used to correct their distortion and estimate two deformation fields (one for $\mathrm{AP} / \mathrm{PA}$ and one for LR/RL). For this specific evaluation, they were applied independently to the spatial landmark positions in AP, PA, LR and RL giving 4 corrected images. If the distortion correction is perfect, the transformed points should then match after transformation. We evaluate this match by computing an average of the one to one Euclidean distances between the landmarks:

$$
d_{i}=\frac{1}{6} \sum_{j=1}^{4} \sum_{k>j}^{4}\left\|p_{i, j}-p_{i, k}\right\|
$$

where $p_{i, j}$ denotes the transformed $i$-th landmark on image $j$ (one of the four images with different PEDs, AP, PA, LR and RL). $d_{i}$ is a distance in millimeters characterizing at which point the four images are distorted after correction around the specific locations of the $p_{i, j}$ : the closer $d_{i}$ is to 0 , the closer the four input images are around the i-th landmark. 
2) Brain images evaluation: intensity-based metrics: To compare images from the brain database after correction, we define a similarity measure computed between the two corrected images $\left(C_{A P / P A}\right.$ and $\left.C_{L R / R L}\right)$.

This evaluation similarity measure $(\mathrm{Sim})$ is defined as a sum of local correlation coefficients normalized between two images. To compute this metric only on relevant areas, masks are computed on four images, the two corrected by TOPUP and the two corrected by BM using the brain extraction tool of the FSL package ${ }^{5}$. Then the union of these four masks is used to obtain the global mask $M$ and therefore compute the similarity measure:

$$
\operatorname{Sim}(I, J, \mathcal{M})=\frac{1}{\operatorname{Card}(\mathcal{M})} \sum_{p \in \mathcal{M}} C\left(I_{\mathcal{N}_{p}}, J_{\mathcal{N}_{p}}\right)
$$

where $I_{\mathcal{N}_{p}}$ and $J_{\mathcal{N}_{p}}$ are neighborhoods of $p$ in $I$ and $J$, defined as a cube centered on $p$ of size $(2 q+1)^{3}$, in the result part $q=3 . C$ is the local correlation between $I_{\mathcal{N}_{p}}$ and $J_{\mathcal{N}_{p}} \cdot \operatorname{Card}(\mathcal{M})$ denotes the cardinal of the set to ensure that Sim belongs to the range $[0,1]$.

Sim characterizes well if the images match after correction and is defined between 0 and 1 which is useful to keep the same stable index between several subjects.

\section{Results}

\section{A. Results on the Phantom}

We first compare the different distortion correction algorithms on the phantom acquisition. We present in Fig. 3 a visual example of the phantom images before and after correction. The BM corrected images are really close and appear visually as being well corrected for distortion. TOPUP images are also properly corrected. Phantom

\footnotetext{
${ }^{5}$ http://fsl.fmrib.ox.ac.uk/fsl/fslwiki/FSL
}

images however do not represent a realistic anatomy and the distortion correction quality may vary depending on the methods. For example, Voss method is not adapted for this kind of bicolor images and gives visually poorer results.

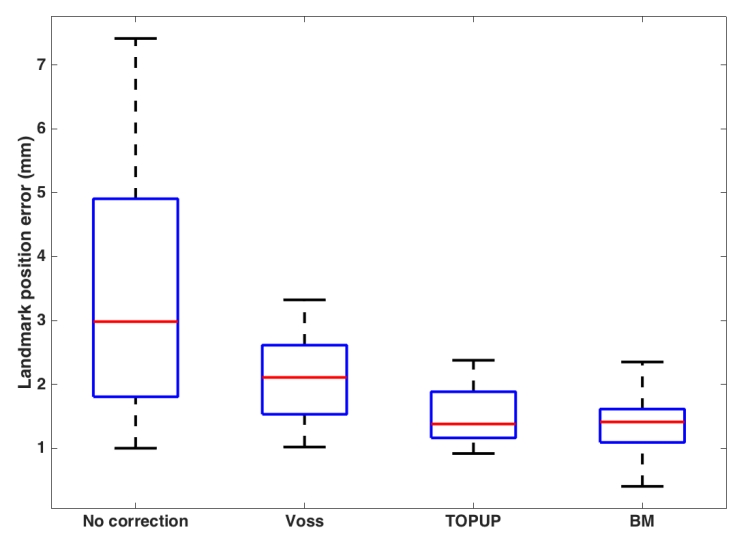

Fig. 4. Landmark position errors on the phantom. Errors (in $\mathrm{mm}$ ) box-plot before and after distortion correction with the Voss correction method, TOPUP and the proposed technique. Error differences are all significant (paired t-test see text for more details).

In addition to visual inspection, we computed landmark position errors based on the distance presented in Section III-C1, Eq. (13). These results are illustrated in Fig. 4 , showing the box-plots of $d_{i}$ distances over all $i$. Voss performs slightly and significantly better than uncorrected images (paired t-test, $p=3 \times 10^{-3}$, average error of $2.09 \mathrm{~mm}$ compared to $3.46 \mathrm{~mm}$ untouched), illustrating its modest performance on this specific dataset. TOPUP also significantly reduces the distance errors, to an average of $1.54 \mathrm{~mm}$, with respect to both uncorrected images (paired

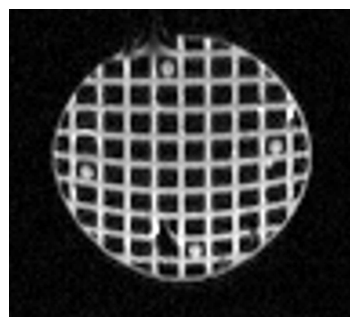

(a) Anterior-Posterior

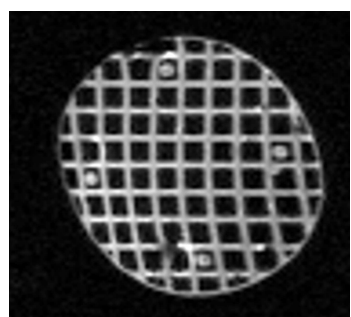

(f) Left-Right

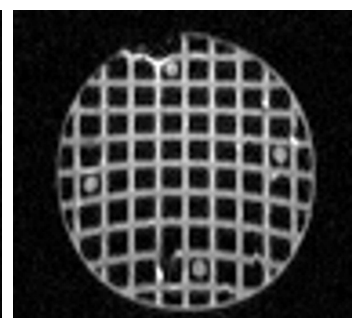

(b) Posterior-Anterior

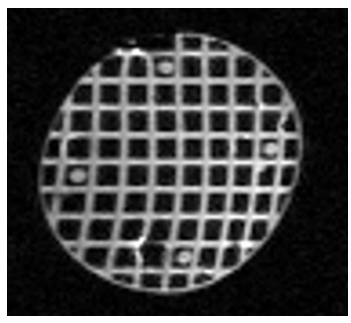

(g) Right-Left

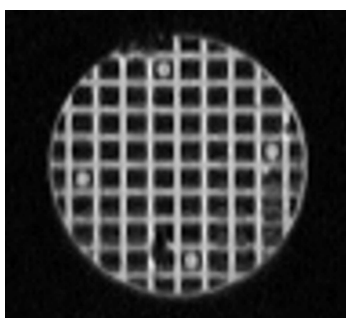

(c) Voss

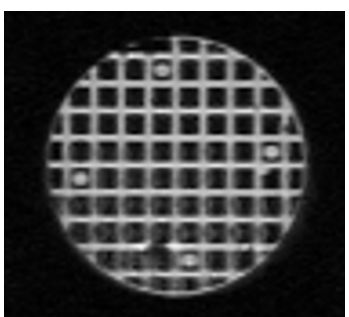

(h) Voss

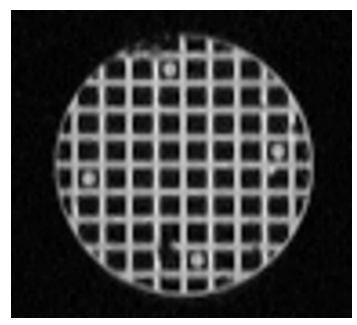

(d) TOPUP

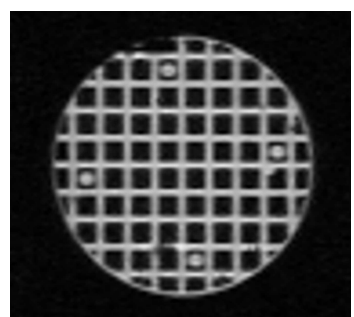

(i) TOPUP

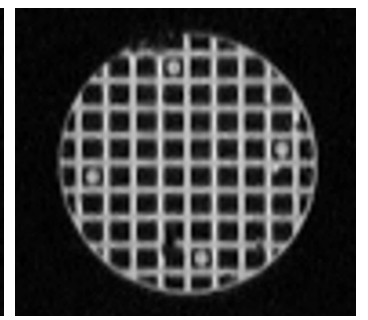

(e) BM

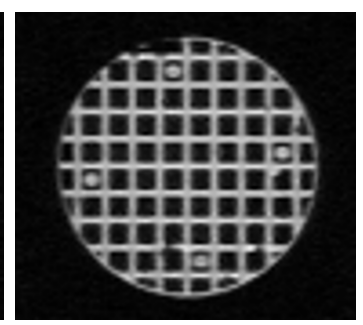

(j) BM

Fig. 3. Distortion correction results on a phantom. Illustration of EPI distortion corrections with the evaluated methods on images acquired with opposite PEDs along the anterior-posterior axis (first line) and the left-right axis (second line). (a-b, $\mathrm{f}$-g): uncorrected $b_{0}$ images, $(\mathrm{c}, \mathrm{h})$ : correction with Voss et al. method, (d,i): correction with TOPUP, (e,j): correction with BM algorithm (proposed technique). 


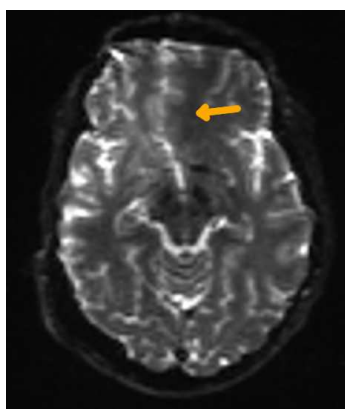

(a) Left-Right $b_{0}$

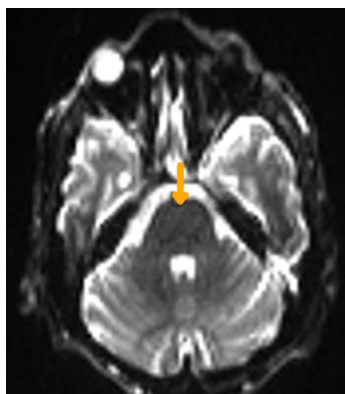

(f) Anterior-Posterior $b_{0}$

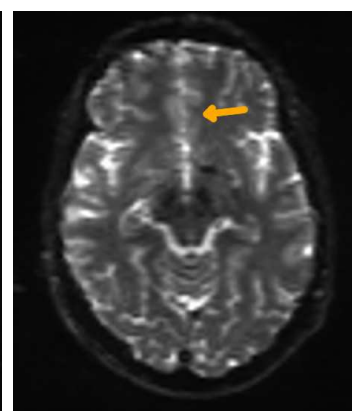

(b) Block-Matching $b_{0}$

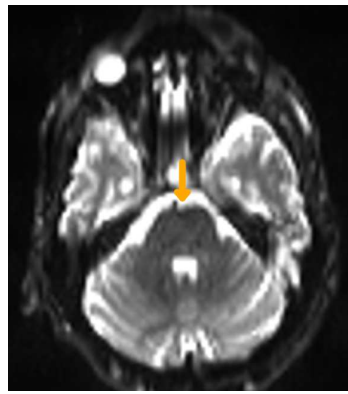

(g) Block-Matching $b_{0}$

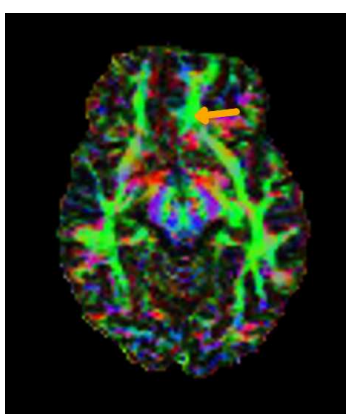

(c) Left-Right FA

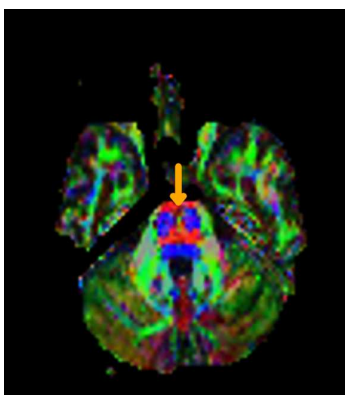

(h) Anterior-Posterior FA

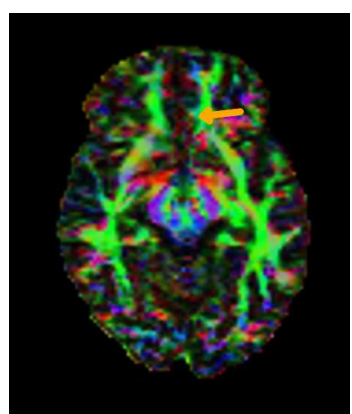

(d) Block-Matching FA

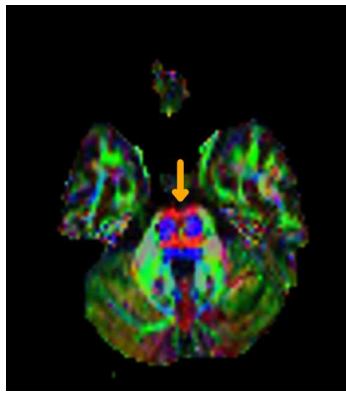

(i) Block-Matching FA

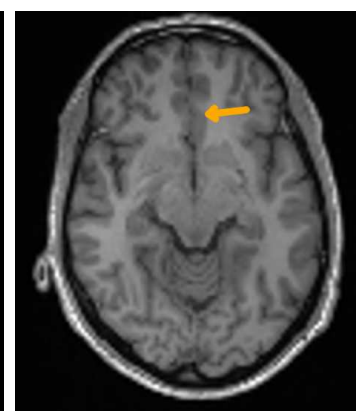

(e) $\mathrm{T} 1$

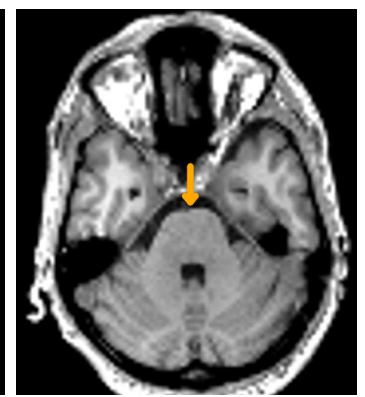

(j) $\mathrm{T} 1$

Fig. 5. Block-Matching distortion correction results compare to original images. Illustration of Block-Matching EPI distortion corrections on $b_{0}$ images acquired with opposite PEDs. The distortion field is computed from the two $b_{0}$ images and applied to an entire DWI volume with a left-right PED for the first line and a anterior-posterior PED for the second one. The fractional anisotropy (FA) is then estimated from the original and the corrected DWI. (a-f): uncorrected $b_{0}$ images, (b,g): block-matching corrected $b_{0}$ images, $(\mathrm{c}, \mathrm{h})$ : uncorrected FA, (d,i): block-matching corrected FA, (e, j): structural T1 reference.

t-test, $p=3 \times 10^{-4}$ ) and to Voss algorithm (paired ttest, $\left.p=1 \times 10^{-3}\right)$. Finally, BM outperforms all other methods obtaining an average error of $1.38 \mathrm{~mm}$, significantly different from uncorrected images (paired t-test, $p=2 \times 10^{-4}$ ), Voss algorithm (paired t-test, $p=4 \times 10^{-4}$ ) and TOPUP (paired t-test, $p=0.028$ ), although both algorithms obtain close precision results, below the voxel size, and are therefore comparable. Overall, these results confirm the visual results, showing that both $\mathrm{BM}$ and TOPUP achieve the best results with BM having the lowest distance error.

\section{B. Results on in-vivo Data}

1) Qualitative Results: We first present in Fig. 5 results of our distortion correction method by visualizing $b_{0}$, corresponding color fractional anisotropy (CFA) images and their corresponding T1 (not distorted) image. The color in CFA map depends on the principal direction of the tensor (red is Left/Right, green Anterior/Posterior and blue Foot/Head) and the intensity of the color is proportional to the FA value. The two lines correspond to a different phase encoding orientation to show distortion in different orientations. It can clearly be seen on Fig. 5 that left-right PED images suffer from large deformations around the falx cerebri (see arrows on Fig. 5.a,c), and on anterior-posterior acquisition the brainstem has a spatial displacement (see arrows on Fig. 5.f,h). On the contrary, our distortion correction method allows for a good matching of the structures in the T1 image and both $b_{0}$ and color FA images. Such a good correction will then allow, for example, for a better definition of regions of interest from the T1 image to seed fiber tracts on the diffusion image, or to study diffusion model properties in specific anatomical regions.

We then illustrate on Fig. 6 distortion correction results of the different evaluated methods on a pair of EPI with opposite PED (anterior-posterior and posterior-anterior). On this strongly affected area, we compared the three different distortion correction methods with respect to a structural image (T1 image). We first noticed that the three corrected images are more similar to the structural image than the original ones, suggesting that each method is able to strongly reduce the distortions. However the image corrected using Voss et al. method (Fig. 6.d) still presents a mismatch around the lateral ventricles (see arrow on Fig. 6.b). TOPUP and BM both obtain a corrected image very close to the structural T1 image.

2) Quantitative Results: We performed experiments on an Intel Xeon 2.5 Ghz computer on 20 cores. The mean time per subject is very short (about $5 \mathrm{~s}$ ) for the Voss algorithm, 170s for the BM and 500s for TOPUP. Unlike TOPUP, BM is multi-threaded, allowing a faster computation time which may be useful in the clinic.

To obtain a quantitative evaluation of the quality of the corrected images, the similarity metric (Sim) introduced in Section III-C2 was computed on the dataset of 5 subjects after correction by the different methods. The Sim metric results between the two corrected images $C_{A P / P A}$ 


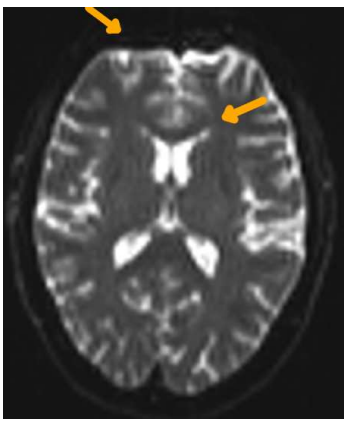

(a) Anterior-Posterior $b_{0}$

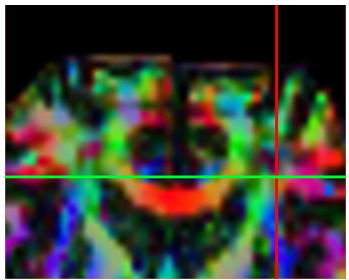

(f) Anterior-Posterior FA

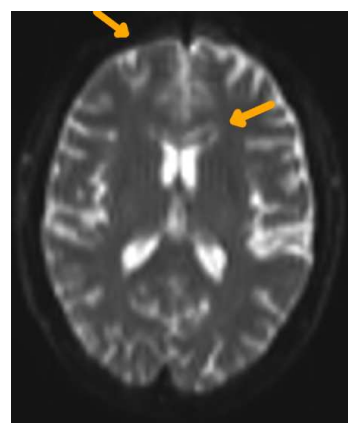

(b) Voss $b_{0}$

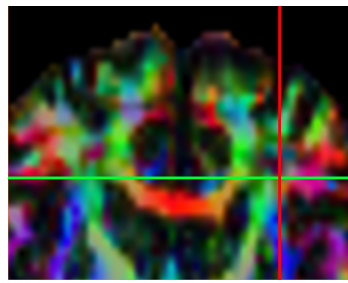

(g) Voss FA

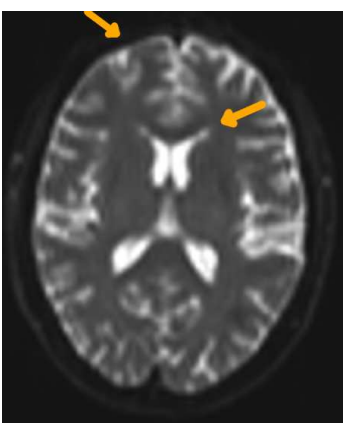

(c) TOPUP FA

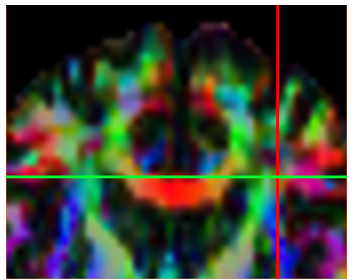

(h) TOPUP FA

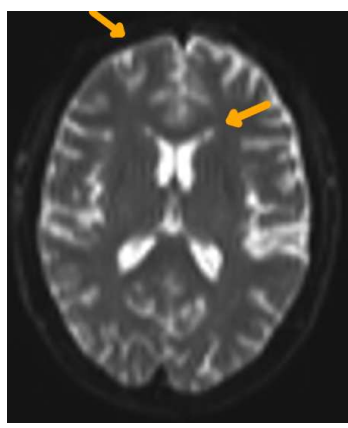

(d) BM FA

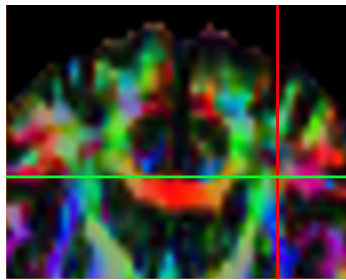

(i) $\mathrm{BM} F A$

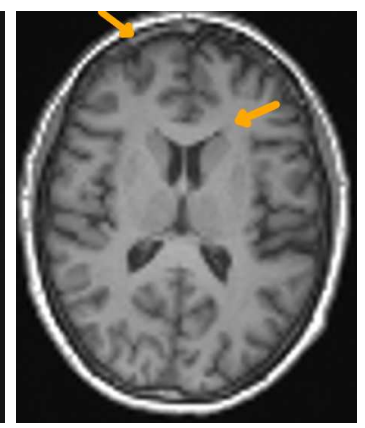

(e) $\mathrm{T} 1$

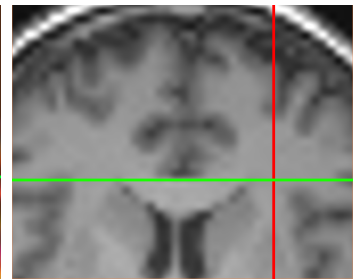

(j) T1

Fig. 6. Registration results on a subject according different distortion correction methods. First row : (a) $b_{0}$ acquired with anterior-posterior PED; Mean of opposed PEDs corrected images : (b) by Voss; (c) by TOPUP; (d) by BM; (e) T1 structural reference. Second row (f) to (i) : zoom on the lateral ventricles of the corresponding colored FA; (j) T1 zoomed.

and $C_{L R / R L}$ are presented in Table I. These results are consistent with visual inspection and highlight that BM performs better than Voss et al. on all subjects, showing a significant improvement of the correction compared to the initialization (Wilcoxon signed-rank test, $p=0.03$ ). Between BM and TOPUP, the best score depends on the subject, the average similarity for the 5 subjects is better for BM than TOPUP however it is not statistically significant (Wilcoxon signed-rank test, $p=0.69$ ).

TABLE I

Correlation results (Sim measure) Between AP/PA and LR/RL IMAGES. COLUMNS FROM LEFT TO RIGHT: NO CORRECTION,

$$
M_{1}, M_{2} \text { AND } M_{3}
$$

\begin{tabular}{|c|c|c|c|c|}
\hline Sim & Untouched & Voss & BM & TOPUP \\
\hline Subject 1 & 0.842 & 0.901 & 0.916 & 0.927 \\
\hline Subject 2 & 0.818 & 0.904 & 0.918 & 0.937 \\
\hline Subject 3 & 0.812 & 0.875 & 0.894 & 0.859 \\
\hline Subject 4 & 0.886 & 0.923 & 0.939 & 0.954 \\
\hline Subject 5 & 0.872 & 0.913 & 0.921 & 0.898 \\
\hline Mean & 0.852 & 0.903 & 0.918 & 0.915 \\
\hline
\end{tabular}

\section{Discussion And Conclusion}

We have presented a new block-matching based algorithm for EPI distortion correction using an additional EPI with reversed PED. To this end, we have developed specific linear transformations constrained to fit a priori with the distortion model at the block level. This transformation definition, integrated into a symmetric blockmatching algorithm, ensures a robust computation of an opposite symmetric transformation.
We have tested our distortion correction and two stateof-the-art methods on a phantom with a known ground truth shape. Our results perform significantly better than Voss et al. correction, which is not adapted for images with uniform intensity regions. Moreover BM performs significantly better than TOPUP algorithm but at a sub-voxel level (though one should not over interpret this difference). Then, we have evaluated the BM registration on 5 subjects with 2 pairs of $b_{0}$ EPI. A similarity measure based on local correlation between the 2 corrected images $C_{A P / P A}$ and $C_{L R / R L}$ show a significant improvement between the Voss initialization and the BM correction. TOPUP algorithm and BM obtain similar levels of similarity. Our BM algorithm is implemented in a multi-threaded fashion using ITK allowing for faster computation time than TOPUP.

A common problem with reversed PED methods is motion since the a priori distortion model is not true any more in that case. The best way to avoid motion problems is to perform an acquisition with continuously alternating PEDs and to correct all pairs independently. For clinical use it is also possible to acquire only one PA and a series of $\mathrm{AP}$ and then correct the entire series from the deformation field estimated. This is more subject to the motion issue but also reduces the acquisition time, which is crucial.

The intensity of distortion in EPI is related to susceptibility-induced fields and eddy current-induced fields. The general trend to increase the scanner field strength increases distortions [33]. Thus it is essential to have efficient tools to correct these distortions. With that goal, we proposed a new simple and robust method, computationally efficient, ready for a clinical use. We studied its application for diffusion MRI, however it can be used for other modalities based on EPI acquisitions such 
as functional MRI.

\section{ACKNOWLEDGMENTS}

Renaud Hédouin PhD is partially funded by the Brittany Council. MRI data acquisition was supported by the Neurinfo MRI research facility from the University of Rennes I. Neurinfo is granted by the the European Union (FEDER), the French State, the Brittany Council, Rennes Metropole, Inria, Inserm and the University Hospital of Rennes. The grid phantom was developed by the UNIRS group from the CEA Neurospin for the CATI Consortium for image acquisition and processing.

\section{REFERENCES}

[1] U. Ferizi, T. Schneider, E. Panagiotaki, G. Nedjati-Gilani, H. Zhang, C. A. M. Wheeler-Kingshott, and D. C. Alexander, "A ranking of diffusion MRI compartment models with in vivo human brain data," Magnetic Resonance in Medicine, vol. 72 no. 6 , pp. $1785-1792,2014$

[2] H. Zhang, T. Schneider, C. A. Wheeler-Kingshott, and D. C. Alexander, "NODDI: Practical in vivo neurite orientation dispersion and density imaging of the human brain," NeuroImage vol. 61, no. 4, pp. 1000 - 1016, 2012.

[3] A. Stamm, P. Pérez, and C. Barillot, "A new multi-fiber model for low angular resolution diffusion MRI," in Biomedical Imaging (ISBI), 2012 9th IEEE International Symposium on, May 2012, pp. 936-939.

[4] M. Taquet, B. Scherrer, O. Commowick, J. M. Peters, M. Sahin, B. Macq, and S. K. Warfield, "A Mathematical Framework for the Registration and Analysis of Multi-Fascicle Models for Population Studies of the Brain Microstructure," IEEE Transactions on Medical Imaging, vol. 2, no. 33, pp. 504-17, 2014.

[5] H. Johansen-Berg and T. Behrens, Diffusion MRI: From quantitative measurement to in-vivo neuroanatomy. Academic Press, 2009.

[6] S. A. Huettel, A. W. Song, and G. McCarthy, Functional magnetic resonance imaging. Sinauer Associates Sunderland, 2004 vol. 1.

[7] P. Jezzard and R. S. Balaban, "Correction for geometric distortion in echo planar images from $\mathrm{B} 0$ field variations," $M R M$ vol. 34 , no. 1 , pp. $65-73,1995$.

[8] P. Reber, E. Wong, R. Buxton, and L. Frank, "Correction of off resonance-related distortion in echo-planar imaging using epibased field maps," Magnetic Resonance in Medicine, vol. 39 no. 2, pp. 328-330, 1998 .

[9] D. Holland, J. M. Kuperman, and A. M. Dale, "Efficient correction of inhomogeneous static magnetic field-induced distortion in echo planar imaging," Neuroimage, vol. 50, no. 1, pp. 175-183, 2010.

[10] M. Wu, L.-C. Chang, L. Walker, H. Lemaitre, A. Barnett, S. Marenco, and C. Pierpaoli, "Comparison of EPI distortion correction methods in diffusion tensor MRI using a novel framework," in MICCAI, Part II, ser. LNCS, vol. 5242, 2008, pp. 321329.

[11] M. Robson, J. Gore, and R. Constable, "Measurement of the point spread function in MRI using constant time imaging," Magnetic Resonance in Medicine, vol. 38, no. 5, pp. 733-740, 1997.

[12] J. chung, M. In, S. Oh, M. Zaitsev, O. Speck, and Z. Cho, "An improved PSF mapping method for EPI distortion correction in human brain at ultra high field (7T)," MAGMA, vol. 24 , no. 3 , pp. 179-190, 2011.

[13] M. Zaitsev, J. Hennig, and O. Speck, "Point spread function mapping with parallel imaging techniques and high acceleration factors: Fast, robust, and flexible method for echo-planar imaging distortion correction," Magnetic Resonance in Medicine, vol. 52 , no. 5 , pp. $1156-1166,2004$.

[14] H. Chang and J. M. Fitzpatrick, "A technique for accurate magnetic resonance imaging in the presence of field inhomogeneities," IEEE Transactions on medical imaging, vol. 11, no. 3, pp. 319-329, 1992.
[15] R. Bowtell, D. McIntyre, M. Commandre, P. Glover, and P. Mansfield, "Correction of geometric distortion in echo planar images," in Soc. Magn. Res. Abstr, vol. 2, 1994, p. 411.

[16] J. L. Andersson, S. Skare, and J. Ashburner, "How to correct susceptibility distortions in spin-echo echo-planar images: application to diffusion tensor imaging," Neuroimage, vol. 20, no. 2, pp. 870-888, 2003.

[17] H. U. Voss, R. Watts, A. M. Uluğ, and D. Ballon, "Fiber tracking in the cervical spine and inferior brain regions with reversed gradient diffusion tensor imaging," Magnetic resonance imaging, vol. 24 , no. 3 , pp. $231-239,2006$.

[18] P. Morgan, R. Bowtell, D. McIntyre, and B. Worthington, "Correction of spatial distortion in EPI due to inhomogeneous static magnetic fields using the reversed gradient method," Journal of Magnetic Resonance Imaging, vol. 19, no. 4, pp. 499-507, 2004.

[19] N. Weiskopf, U. Klose, N. Birbaumer, and K. Mathiak, "Singleshot compensation of image distortions and BOLD contrast optimization using multi-echo EPI for reltime fMRI," Neuroimage, vol. 24, no. 4, pp. 1068-1079, 2005.

[20] M.-H. In, O. Posnansky, E. B. Beall, M. J. Lowe, and O. Speck, "Distortion correction in EPI using an extended psf method with a reversed phase gradient approach," PloS One, vol. 10, no. 2, 2015.

[21] M. Irfanoglu, P. Modi, A. Nayak, E. Hutchinson, J. Sarlis, and C. Pierpaoli, "DR-BUDDI (diffeomorphic registration for blipup blip-down diffusion imaging) method for correcting echo planar imaging distortions," Neuroimage, vol. 106, pp. 284-299, 2015.

[22] U. Vovk, F. Pernus, and B. Likar, "A review of methods for correction of intensity inhomogeneity in mri," IEEE transactions on medical imaging, vol. 26, no. 3, pp. 405-421, 2007.

[23] S. Ourselin, A. Roche, S. Prima, and N. Ayache, "Block matching: A general framework to improve robustness of rigid registration of medical images," in Medical Image Computing and Computer-Assisted Intervention-MICCAI 2000. Springer, 2000, pp. 557-566.

[24] O. Commowick, N. Wiest-Daesslé, and S. Prima, "Automated diffeomorphic registration of anatomical structures with rigid parts: Application to dynamic cervical MRI," in Medical Image Computing and Computer-Assisted Intervention-MICCAI 2012. Springer, 2012, pp. 163-170.

[25] O. Commowick, N. Wiest-Daesslé, and S. Prima, "Blockmatching strategies for rigid registration of multimodal medical images," in 2012 9th IEEE International Symposium on Biomedical Imaging (ISBI). IEEE, 2012, pp. 700-703.

[26] B. B. Avants, C. L. Epstein, M. Grossman, and J. C. Gee, "Symmetric diffeomorphic image registration with cross-correlation: evaluating automated labeling of elderly and neurodegenerative brain," Medical image analysis, vol. 12, no. 1, pp. 26-41, 2008.

[27] P. J. Burt and E. H. Adelson, "The laplacian pyramid as a compact image code," Communications, IEEE Transactions on vol. 31, no. 4, pp. 532-540, 1983.

[28] M. J. Powell, "The BOBYQA algorithm for bound constrained optimization without derivatives," Centre for Mathematical Sciences, University of Cambridge, UK, Tech. Rep., 2009.

[29] C.-F. Westin, S. E. Maier, H. Mamata, A. Nabavi, F. A. Jolesz, and R. Kikinis, "Processing and visualization for diffusion tensor MRI," Medical image analysis, vol. 6, no. 2, pp. 93-108, 2002.

[30] V. Arsigny, O. Commowick, N. Ayache, and X. Pennec, "A fast and log-euclidean polyaffine framework for locally linear registration," Journal of Mathematical Imaging and Vision, vol. 33, no. 2, pp. 222-238, 2009 .

[31] T. Vercauteren, X. Pennec, A. Perchant, and N. Ayache, "Symmetric log-domain diffeomorphic registration: A demons-based approach," in Medical Image Computing and Computer-Assisted Intervention - MICCAI 2008, ser. Lecture Notes in Computer Science, vol. 5241, 2008, pp. 754-761.

[32] I. J. Schoenberg, Cardinal spline interpolation, ser. Regional conference series in applied mathematics. Philadelphia, Society for Industrial and Applied Mathematics, 1973, vol. 12.

[33] F.-N. Wang, T.-Y. Huang, F.-H. Lin, T.-C. Chuang, N.-K. Chen, H.-W. Chung, C.-Y. Chen, and K. K. Kwong, "Propeller epi: an mri technique suitable for diffusion tensor imaging at high field strength with reduced geometric distortions," Magnetic resonance in medicine, vol. 54, no. 5, pp. 1232-1240, 2005. 